\title{
Genomic Responses Associated with Drought Stress in Walnut as Revealed Transcriptome Sequencing
}

Mohammad Sadat-Hosseini

University of Tehran

Mohammad Reza Bakhtiarizade

University of Tehran

Naser Boroumand

Shahid Bahonar University of Kerman

Masoud Tohidfar

Shahid Beheshti University

Sasan Aliniaeifard

University of Tehran

Kourosh Vahdati ( $\boldsymbol{D}$ kvahdati@ut.ac.ir)

University of Tehran

\section{Research Article}

Keywords: Abiotic stress, differentially expressed genes, Drought, Gene, Juglans regia, Transcriptome, Water deficit.

Posted Date: December 13th, 2021

DOI: https://doi.org/10.21203/rs.3.rs-1006467/v1

License: (c) (1) This work is licensed under a Creative Commons Attribution 4.0 International License. Read Full License 


\section{Abstract}

Walnut production is challenged by abiotic stresses. We investigated the leaf transcriptome responses of walnut under control and drought stress in 9 and 18 days. We identified 921, 1035 differentially expressed genes (DEGs) between control and drought stress groups in 9 and 18-day, respectively. In control and drought stress conditions DEGs were significantly enriched into the abscisic acid biosynthesis, regulation of stomata closure, leaf morphogenesis, carbohydrate metabolism, oxidative stress, cell wall macromolecule catabolism, and secondary metabolite biosynthesis pathways. We confirmed our RNA-Seq data using quantitative real-time PCR (qPCR) of six candidate genes. Our results indicated that more complicated transcript regulation of drought responses following prolong exposure to drought stress. In general, walnut activated more tolerance mechanisms 18 days after drought stress. Findings of this research would be useful for future studies on breeding for drought tolerance of Persian walnut and related species.

\section{Introduction}

Persian or English walnut (Juglans regia L.) is native to the central Asia, but is now distributed in worldwide in temperate areas for nut production ${ }^{1}$. The highest walnut production in the world belongs to the United States, China and Iran, respectively. From a nutritional point of view, walnuts provide appreciable amounts of proteins, carbohydrates and are rich in unsaturated fatty acids including, linoleic, linolenic acids and oleic ${ }^{1}$. In a large part of the areas where walnuts are produced, researchers have discovered that drought stress severely reduced yield ${ }^{2-4}$. In the current changing global climate scenario, water availability is one of the most limiting factors that determine plant distribution and productivity. Responses of plants to drought stress may occur from cell membrane to photosynthetic organs, to the whole plants ${ }^{5}$. Drought stress in plants is a complex phenomenon that triggers diverse modifications from gene to morphology ${ }^{6}$. To survive, plant provides physiological (e.g. stomatal movements, ABA signaling, membrane transport, osmolyte regulation), biochemical (enzyme and non-enzyme activity), morphological (changes in leaf and root growth, wilting of leaves) and molecular (changing the expression of a number of genes, transcription factors and micro-RNAs) responses ${ }^{7,8}$. Hence, comprehensive surveys of mechanisms by which walnuts are affected by drought stress are needed, especially in arid and semi-arid regions. Thanks to next-generation sequencing (NGS)-based technologies, gene expression profiling using RNA sequencing method has become a highly sensitive and accurate method, which can be applied to study the plant responses to biotic and abiotic stresses ${ }^{9}$. Recently, many studies have been conducted to understand the molecular mechanisms of drought tolerance using comparative transcriptome by RNA-Seq in many plant species such as tomato ${ }^{10}$, sorghum ${ }^{9}$, $\operatorname{cotton}{ }^{11}$, camellia ${ }^{12}$, pear ${ }^{13}$, peach ${ }^{14}$, eucalyptus ${ }^{15}$, California oak ${ }^{6}$, and Jhar Ber ${ }^{16}$. In spite of drought stress importance, most of the studies considered morphological and physiological indices and no studies have been investigated the molecular mechanisms (including gene expression profiling) in response of walnut to drought stress. A study reported that seed germination of walnut in drought stress conditions significantly reduced and soluble sugars and proline accumulate in shoots and roots in tolerant 
genotypes ${ }^{17}$. It has been also reported that petioles loss $87 \%$ of their maximum hydraulic conductivity in drought-exposed walnuts, causing leaf abscission ${ }^{18}$. In spite of these studies, the molecular mechanisms underlying drought stress on walnut remains largely unknown and needs to be further investigated. Therefore, in this study, RNA-Seq technology was used to understand the genome-wide transcriptome changes of walnut following mid and long-term exposure to drought stress condition and to characterize differential physiological response of walnut under normal and drought conditions.

Results of this study will expand the knowledge of IncRNAs participating drought response in walnut and may be applied to improve productivity of walnuts under drought stress.

\section{Results}

\section{Effect of mid- and long-term drought stress on physiological parameters of walnut plants}

Overall, drought stress had significant effects $(P<0.01)$ on RWC, SPAD index and MSI at both 9th and 18th days of drought stress exposure. RWC in control plants was $81.2 \%$ and $80.23 \%$ at 9 th and 18 th $d$ respectively, but under drought stress this index reduced by $61.1 \%$ and $48.7 \%$ at 9 th and 18 th $\mathrm{d}$. Also, SPAD index reduced in water deficit-exposed plants from 38 to 30.3 at day 9th and 37.2 to 23.4 at day 18th of drought stress exposure. Finally, MSI was reduced by drought stress conditions $(35.4 \%$ and $31.1 \%$ at 9 and 18 d) than control ( $57.3 \%$ and $53.6 \%$ at 9 th and 18 th d) (Table 1 ).

Table 1

change in RWC, SPAD and MSI in control and drought stress of walnut in 9th and 18th $d$ after the start of experiment

\begin{tabular}{|lllllll|}
\hline & RWC (\%) & & SPAD & \multicolumn{3}{c|}{ MSI (\%) } \\
\hline Control & $\begin{array}{l}\text { Drought } \\
\text { stress }\end{array}$ & Control & $\begin{array}{l}\text { Drought } \\
\text { stress }\end{array}$ & Control & $\begin{array}{l}\text { Drought } \\
\text { stress }\end{array}$ \\
\hline 9 d & $\mathbf{8 1 . 2}$ & 61.1 & $\mathbf{3 8}$ & $\mathbf{3 0 . 3}$ & $\mathbf{5 7 . 3}$ & $\mathbf{3 5 . 4}$ \\
\hline $18 \mathrm{~d}$ & 80.23 & 48.7 & 37.2 & 23.4 & 53.6 & 31.1 \\
\hline Significant & $* *$ & & $* *$ & & $* *$ & \\
\hline
\end{tabular}

At 18th $\mathrm{d}$ of drought stress exposure parameters related to fluorescence of chlorophyll were measured to evaluate the healthiness of photosynthesis apparatus of plants. Some of indexes such as Fv/Fm, $\mathrm{ABS} / \mathrm{RC}, \mathrm{PI} A B S$, Dlo/RC and Fv/Fo affected under drought conditions (Fig. 1). The parameter related to electron transport and functionality of the photosynthesis apparatus including Fv/Fm, PI ABS and Fv/Fo 
decreased in the plants under drought stress, simultaneous ABS/RC and Dlo/RC parameter that associated to dissipation of energy increased under drought stress.

\section{Rna Sequencing And Assembly}

The plants were kept under control and drought stress conditions for 18th d. Leaf sampling was performed at two time points, 9th (C9 and S9) and 18th (C18 and S18) d after the start of the experiment, and three biological replications were conducted for each group. In total, 240 million paired-end reads were generated of 11 libraries with an average of 21.8 million reads per library. After removing the adapters, low-quality reads and correction of errors, 231 million clean reads were remained for further analysis (Table S1). Our de novo assembly pipeline, based on various quality metrics of assembly, showed that the transcriptome assembly produced by EvidentialGene can be prioritized over other assemblies in walnut leaves. The reference transcriptome assembly generated by EvidentialGene included 183,191 transcripts with an N50 length of 1,831 bp, among which 64,702 transcripts were longer than $1 \mathrm{~kb}$ (Table S2).

\section{Functional Annotation}

All the assembled transcripts were analyzed and compared against public databases (NCBI nr database, UniProtKB database, Pfam database, Rfam database, miRbase database, PInTFDB database, SignalP software, Rnammer software and tmHMM software) by different tools including BLAST, HMMER, TransDecoder, RNAMMER v1.2, TMHMM v2.0, SignalP v4.1, and KASS. The results revealed that $61 \%$ (111,451 transcripts) of 183,191 transcripts were annotated using one or more databases. Briefly, a total of $104,926(57.27 \%)$ ORFs, 79,185 (43.22\%) transcripts with at least one predicted domain, 3,931 (2.14\%) transcription factor, 6,591 (3.59\%) signal peptides, and 92,704 (50.6\%) transmembrane domains were identified. This analysis identified $109,413(59.72 \%)$ and $84,384(46.06 \%)$ hits from the NCBI $\mathrm{nr}$ and the UniProtKB databases, respectively. In order to better comprehend the biological pathways in the transcripts, the annotated sequences were analyzed by KEGG database. Results of pathway analysis showed that all the transcripts were classified to five specific pathways including Organism Systems, Cellular Processes, Environmental Information Processing, Genetic Information Processing and Metabolism (Fig. 2). The largest number of the transcripts were belonged to Metabolic pathway $(8,050$, $32.58 \%)$ followed by Organism Systems $(5,630,22.79 \%)$, Environmental Information Processing $(3,951$, $15.99 \%)$, Genetic Information Processing $(3,786,15.32 \%)$ and Cellular Processes $(3,284,13.29 \%)$. Moreover, Signal transduction $(3,857,15.68 \%)$ and Carbohydrate metabolism $(2,028,8.21 \%)$ were the top two representative pathways (Fig. 2).

Gene Ontology (GO) classification of all the annotated transcripts were performed based on the three functional groups; biological process (BP), molecular function (MF) and cellular component (CC). Out of 183,191 transcripts, 81,494 transcripts were contained at least one GO term (cellular compounds $(69,717$ transcripts), molecular functions (70,689 transcripts) and biological processes (64,992 transcripts)). All 
the annotated transcripts were classified to 56 functional categories using WEGO plot (Fig. 3). In the biological process category, most of the transcripts were related to 'cell', 'cell part', 'organelle' 'membrane part' and 'protein-containing complex'. Also, 'catalytic activity', 'binding', 'transcription regulator activity' and transporter activity' had highest percentage in the molecular function category. Finally, in the biological process category 'cellular process', 'metabolic process', 'biological regulation', 'response to stimulus' and 'regulation of biological process' were the most representative categories. A number of organelle plants including cell, cell part and membrane part are affected at the beginning of drought stress.

\section{Identified DEGs between control (C9) and drought stress (S9) at 9th d}

The analysis showed that 921 genes were DEGs, of which 580 genes were up-regulated and 341 genes were down-regulated in $\mathrm{S} 9$ than $\mathrm{C} 9$ group. Results of the functional enrichment analysis of up-regulated genes revealed 27 significant $\mathrm{GO}$ terms in biological process category including 'secondary metabolite biosynthesis', 'response to oxidative stress', 'carbohydrate metabolism', 'polysaccharide digestion' and 'cell wall macromolecule catabolism' pathways (Fig. 4a). Also, 11 biological process terms were significantly enriched in the down-regulated genes including 'leaf morphogenesis', 'polysaccharide biosynthesis', 'cell wall organization' and 'acetyl-coA biosynthesis' pathways were significant (Fig. 4b). On the other hand, KEGG pathway enrichment analysis revealed four and three significant enriched pathways in up-regulated and down-regulated genes, respectively (Table 2).

Table 2

Important genes for each pathway with fold change represented in walnut under drought stress in 9th d.

\begin{tabular}{|c|c|c|}
\hline & Pathways & Gene name (Fold change) \\
\hline \multirow[t]{4}{*}{$\begin{array}{l}\text { Up- } \\
\text { regulated }\end{array}$} & Oxidative stress & $\begin{array}{l}\text { BCB (14.2), SIP2 (10.49), CYC6 (9.34), APXS (9.16), PXD } \\
\text { (5.3) and OSA1 (2.19) }\end{array}$ \\
\hline & Carbohydrate metabolism & $\begin{array}{l}\text { MDI (9.9), BGAL1 (8.3), R1 (4.03), Lichenase (2.4) and } \\
\text { HGN1 (2.4) }\end{array}$ \\
\hline & $\begin{array}{l}\text { Cell wall macromolecule } \\
\text { catabolism }\end{array}$ & HSP7P (10.77), KTI2 (3.96) and CALS3 (11.41) \\
\hline & $\begin{array}{l}\text { Secondary metabolite } \\
\text { biosynthesis }\end{array}$ & $\begin{array}{l}\text { CYP71 (6.46), MO3 (4.54), CYP89 (3.66) and LKR/SDH } \\
(2.25)\end{array}$ \\
\hline \multirow[t]{3}{*}{$\begin{array}{l}\text { Down- } \\
\text { regulated }\end{array}$} & Leaf morphogenesis & $\begin{array}{l}\text { TCP4 (1.64), GAE6 (1.95), LNG1 (2.09), TCP2 (3.31) and } \\
\text { LUH (7.5) }\end{array}$ \\
\hline & $\begin{array}{l}\text { Polysaccharide } \\
\text { biosynthesis }\end{array}$ & GATL9 (9.32), AXS2 (1.5), GAE6 (1.95) and GATL9 (2.65) \\
\hline & Acetyl-coA biosynthesis & ACS (4.4) \\
\hline
\end{tabular}




\section{Identified DEGs between control (C18) and drought stress (S18) at 18th d}

A total of 1035 DEGs were identified between the control and drought stress group in 18 day after the start of experiment $(P<0.05)$. Of these, 850 genes were up-regulated, while 180 genes were downregulated in S18 than C18 group. GO enrichment analysis of up-regulated DEGs showed that the 'response to drought stress', 'abscisic acid', 'secondary metabolite biosynthesis', 'response to salicylic acid', 'response to oxidative stress', 'response to salt stress', 'response to osmotic stress', 'polysaccharide digestion', 'regulation of stomatal opening', 'malate transport' and 'carbohydrate metabolism' pathways were enriched (Fig. 5a). Likewise, in the down-regulated DEGs 'photosystem $\triangle$ stabilization', 'ATP biosynthesis', 'negative regulation of response to water deprivation', 'vacuole organization' and 'starch catabolism' pathways were significant terms (Fig. 5b). KEGG pathway analysis predicted the involvement of up-regulated and down-regulated DEGs in seven and three pathways, respectively (Table 3).

Table 3

Important genes for each pathway with fold change represented in walnut under drought stress in 18th d.

\section{Pathways Gene name (Fold change)}

$\begin{array}{lll}\begin{array}{l}\text { Up- } \\ \text { regulated }\end{array} & \begin{array}{l}\text { Response to } \\ \text { drought stress }\end{array} & \text { MPK3 (10.8), MAPKKK17 (6.69), Map3k1 (7.71), ABR1 (4.95), } \\ & \text { RMA1H1 (4.6), EDL3 (4.71), XERICO (3.5), }\end{array}$

Abscisic acid $\quad$ AHG1 (9.58), ARIA (9.26), CCA1 (8.81), NCED6 (6.83), SAG21 (5.14),

ABA4 (4.6), XERICO (3.5), AFP2 (4.35), NCED1 (3.15), MARD1 (2.79),

Secondary metabolite

CYP71B37 (5.91), CYP82A3 (5.42), MO3 (5.8), CYP71B34 (4.62)

biosynthesis

Carbohydrate rhiE (12.1), Chi3l1 (9.26), rfs5 (8.12), GNS1 (5.48), HGN1 (5.13), GN1 metabolism (4.81), NANA (3.7)

Response to MAPK3 (10.83), GATL10 (5.5), SAG21 (5.14), CSE (3.97), HSPRO2 oxidative stress (3.36)

Response to $\quad$ CCA1 (8.81), GLP9 (8.35), SRO5 (7.8), GLYI (4.44), MKK9 (4.11), salt stress $\quad$ SRM1 (3.9)

Regulation of $\quad$ ALIS1 (10.9), IQM1 (8.87), MYB15 (4.92), RZPF34 (4.89), CKX6 (4.6) stomatal opening

\begin{tabular}{|c|c|c|}
\hline $\begin{array}{l}\text { Down- } \\
\text { regulated }\end{array}$ & $\begin{array}{l}\text { ATP } \\
\text { biosynthesis }\end{array}$ & WAK2 (2.79), AHA10 (2.7), BAM3 (2.66), ABCC3 (2.73), AB3C (3.04) \\
\hline
\end{tabular}

vacuole $\quad$ DTX41 (2.33), ABCC3 (2.73), YPQ1 (5.86), MON1 (8.16) organization

starch catabolism

WAXY (2.77), LECRK1 (2.65), BAM3 (2.65) 


\section{Functional KEGG pathways for DEGs in 18 day after the start of experiment}

Since the more pathways were activated 18 days than 9 day, up-regulated pathway enrichment conducted using the KEGG database. There were 20 KEGG pathway recognized as enriched significant with different $p$ value. According to the Table 4. the important pathways that significantly in drought stress conditions including 'Transcription Factors', 'Plant hormone signal transduction', 'Starch and sucrose metabolism', 'Galactose metabolism' and 'Mitogen-activated protein kinase (MAPK) cascade'. Also, the pathway enrichment for down-regulated genes were showed in the Table 5. 
Table 4

Functional analysis up-regulated DEGs based on the KEGG pathways in walnut 18th $\mathrm{d}$ after the beginning of experiment.

\begin{tabular}{|c|c|c|c|}
\hline Enriched pathway term & ID & $\begin{array}{l}\text { Number of } \\
\text { transcripts }\end{array}$ & P-Value \\
\hline Transcription Factors & ko03000 & 20 & 0.02 \\
\hline Plant hormone signal transduction & ko04075 & 18 & 0.00025 \\
\hline Starch and sucrose metabolism & ko00500 & 14 & 0.00056 \\
\hline Galactose metabolism & ko00052 & 12 & $4.18 \times 10^{-7}$ \\
\hline $\begin{array}{l}\text { Mitogen-activated protein kinase (MAPK) } \\
\text { cascade }\end{array}$ & ko04016 & 11 & 0.015 \\
\hline Glycolysis & ko00010 & 9 & 0.032 \\
\hline Tryptophan metabolism & ko00380 & 6 & 0.00012 \\
\hline Isoquinoline alkaloids biosynthesis & ko00950 & 5 & 0.00027 \\
\hline Lysine degradation & ko00310 & 5 & 0.0081 \\
\hline Tyrosine metabolism & ko00350 & 5 & 0.009 \\
\hline Cytochrome P450 & ko00982 & 5 & 0.014 \\
\hline Ascorbate and aldarate metabolism & ko00053 & 5 & 0.015 \\
\hline Alpha-Linolenic acid metabolism & ko00592 & 5 & 0.016 \\
\hline Amino acid metabolism & ko00250 & 5 & 0.018 \\
\hline Glycine, serine and threonine metabolism & ko00260 & 5 & 0.02 \\
\hline Beta-Alanine metabolism & ko00410 & 5 & 0.025 \\
\hline Metabolism; Amino acid metabolism & ko00280 & 5 & 0.025 \\
\hline Histidine metabolism & ko00340 & 3 & 0.036 \\
\hline Betalain biosynthesis & ko00965 & 2 & 0.00018 \\
\hline
\end{tabular}


Table 5

KEGG pathway analysis of down-regulated DEGs between the control and drought stress groups in 18th d after the start of experiment.

\begin{tabular}{|llll|}
\hline Enriched pathway term & ID & $\begin{array}{l}\text { Number of } \\
\text { transcripts }\end{array}$ & P-Value \\
\hline Transporters & ko02000 & 14 & 0.0013 \\
\hline Flavonoid biosynthesis & ko00941 & 13 & $3 \times 10^{-17}$ \\
\hline $\begin{array}{l}\text { Ubiquinone and other terpenoid-quinone } \\
\text { biosynthesis }\end{array}$ & ko00130 & 6 & $3.42 \times 10^{-6}$ \\
\hline Phenylpropanoid biosynthesis & ko00940 & 4 & 0.0031 \\
\hline Glutathione metabolism & ko00480 & 4 & 0.0016 \\
\hline Circadian rhythm & ko04712 & 3 & 0.001 \\
\hline
\end{tabular}

\section{Identified Degs Between Drought Stress (S9) And (S18) Groups}

A comparison was conducted to identify DEGs in drought stress groups at 9th and 18th $\mathrm{d}$ after the start of experiment. Overall, 841 DEGs between S9 and S18 groups were identified, of which 769 genes were up-regulated and 76 genes were down-regulated in S18 than S9 groups. On the basis of G0 analysis 33 biological terms including 'carbohydrate metabolism', 'activation of MAPKK activity', 'response to osmotic stress', 'abscisic acid metabolism', 'proline catabolism' and 'secondary metabolite biosynthesis' were enriched in up-regulated DEGs (Fig. 6a). As displayed in Fig. 6b, down-regulated DEGs were significantly enriched 10 biological terms including 'regulation of photosynthesis', 'stomatal complex morphogenesis' and 'positive regulation of response to oxidative stress'. Fig. 7a and Fig. 7b depicted the number of common and unique up-regulated and down-regulated DEGs in the three performed comparisons (C9-S9, C18-S18 and S9-S18), respectively.

\section{Transcription Factors (Tfs) In Degs}

Because of the importance of TFs in gene expression regulations, especially in response to drought stress, TFs in the DEGs were identified. A total of 28 TFs belonged to 10 families were identified including MYB, NAC, WRKY and ERF families. Most of the identified TFs were belonged to MYB group, as MYB78 and MYB3R1 were up-regulated and showed the highest fold change (10.77 and 9.38 fold change, respectively) (Table 6). 
Table 6

Transcription factors identified in walnut transcriptome under drought stress at 18th $\mathrm{d}$ after the beginning of the experiment.

\begin{tabular}{|llll|}
\hline Transcription factor & Up-regulated (Fold change) & Transcription factor & $\begin{array}{l}\text { Up-regulated } \\
\text { (Fold change) }\end{array}$ \\
\hline MYB78 & 10.77 & NAC029 & 6.01 \\
\hline MYB3R1 & 9.38 & NAC073 & 5.07 \\
\hline MYB2 & 6.03 & NAC072 & 4.83 \\
\hline MYB73 & 5.31 & NAC100 & 3.8 \\
\hline MYB108 & 5.12 & NAC002 & 3.5 \\
\hline MYB15 & 4.9 & ERF1 & 7.99 \\
\hline MYB69 & 4.8 & ERF1B & 5.3 \\
\hline MYB3 & 3.1 & ERF1A & 3.6 \\
\hline WRKY40 & 10.11 & ERF4 & 3.56 \\
\hline WRKY75 & 6.57 & SIB1 & 8.3 \\
\hline WRKY14 & 5.8 & RAP2 & 2.9 \\
\hline WRKY6 & 4.08 & EIN3 & 2.26 \\
\hline ARF5 & 8.8 & EDL3 & 3.27 \\
\hline ARF6 & 3.3 & ASIL2 & 9.19 \\
\hline
\end{tabular}

\section{Validation Of Degs Using Rt-pcr}

Validation of DEGs using RT-PCR

To validate the RNA-seq results, six genes including four up-regulated (RCD1, ALDH, PGI1 and MPK3) and two down-regulated (ERL2 and LECRK1) were randomly picked from DEGs. The ratio of the Log2 fold change from the RNA-seq data was compared to the Log2 fold change obtained with RT-PCR. As shown in Fig. 8, all the six genes displayed similar expression patterns in both RNA-Seq and RT-PCR results, confirming the reliability of the RNA-Seq data.

\section{Discussion}

Drought stress is a multidimensional stress causes diverse modifications including metabolic adjustment, transcriptomic changes, osmotic adjustment, leaf turgor pressure alterations, proteomic 
changes and retardation or deceleration of plant growth ${ }^{19-21}$. In the present study, RWC, SPAD index and MSI were down-regulated by $9^{\text {th }}$ and $18^{\text {th }} \mathrm{d}$ exposure to drought stress condition. These findings are in line with the previous reports that identified down-regulation of physiological parameters by drought stress in Persian walnut $2,17,22,23$. In addition, parameters related to the photosynthesis functionality such as Fv/Fm, PI ABS, Dlo/RC and Fv/Fo related to photosynthesis, were negatively affected by drought stress condition at $18^{\text {th }} \mathrm{d}$ (Fig. 1). These findings confirms occurrence of photoinhibition in water deficitexposed walnut plants. Imbalance between products of light-dependent reactions (ATP and NADPH) and consumption of these products in Calvin-Benson cycle is usually occurred as a result of long-term stomatal limitation for provision of enough $\mathrm{CO}_{2}$ as the substrate for RUBISCO enzyme in Calvin-Benson cycle ${ }^{24,25}$. This problem emerges because there are many enzymatic reactions in Calvin-Benson cycle and they decelerate when they are working under low mesophyll $\mathrm{CO}_{2}$ concentration ${ }^{26}$. Although at the beginning of drought stress exposure there would be no limitation on light-dependent reactions and they are still running and transfer the electrons to $\mathrm{NADP}^{+}$to produce $\mathrm{NADPH}$; after a while the $\mathrm{NADP}^{+}$pool would be limited due to down-regulation of enzymatic reactions in Calvin-Benson cycle. This imposes an imbalance between two sets of the reactions directing the excited electrons toward oxygen and produces reactive oxygen species (ROS) ${ }^{27}$. Reduction in MSI as an indicator for oxidative stress can be related to the damages caused by ROS accumulation. It seems rational to postulate that these results are due to that drought stress induced much more adverse photoinhibitory impairment in drought stress (S18) than control (C18) conditions. Changes in the above indicators indicated that plants were continuously damaged by drought stress in $9^{\text {th }}$ and $18^{\text {th }} \mathrm{d}$.

KEGG pathway enrichment analysis showed that most of transcripts were related to metabolic pathway than the other pathways (Fig. 2). In this regard, carbohydrate metabolism and amino acid metabolism included most of transcripts (2028 and 1170 transcripts, respectively). Previous studies reported that carbohydrate metabolism was significantly enriched under drought stress ${ }^{28-30}$. Moreover, a large number of the transcripts $(3,847)$ were annotated to be participated in signal transduction pathway. Many reports are available in the literature that described rapid responses of plants to drought stress (molecular changes, TFs activation, hormones transmission of hormones and stomatal closing) often mediated by signal transduction pathway ${ }^{29,30}$. According to the $\mathrm{GO}$ enrichment analysis, 'metabolic process, 'response to stimulus', and 'signaling' biological process terms had the most transcripts (Fig. 3). The findings of the current study are consistent with other studies such as in apple ${ }^{31}$, sweet orange ${ }^{32}$ and apricot ${ }^{33}$ which reported that under drought stress 'metabolic process' and 'response to stimulus' are the highest transcripts in biological process category.

Response to oxidative stress was one of the enriched pathways in DEGs in S9 to C9 comparison. Drought condition leads to an imbalance between the production of ROS and the plant's antioxidant defenses, which causes oxidative stress in plants. For example, role of APXS gene, that encode the enzyme ascorbate peroxidase, detoxify hydrogen peroxide $\left[\mathrm{H}_{2} \mathrm{O}_{2}\right]$ as an ROS in plants ${ }^{34}$. Another gene is PXD, which plays important role in peroxidase enzyme synthesis to scavenge $\mathrm{H} 2 \mathrm{O} 2{ }^{35}$. Some of the up- 
regulated genes belong to carbohydrate metabolism pathway have been shown to be up-regulated in drought stress. Carbohydrate helps in stabilizing cellular membranes, keeping the leaf water potential and osmotic adjustment in plants that are subjected to drought stress ${ }^{36}$. Previous studies demonstrated that carbohydrate has important osmotic role for drought tolerance in walnut ${ }^{2,22,23}$. According to the literature, under drought stress secondary metabolites accumulation in plants ${ }^{37}$. In the current study, secondary metabolite biosynthesis pathway activated in drought stress groups. The important gene belonged to secondary metabolite biosynthesis which up-regulated were CYP71, MO3, CYP89 and LKR/SDH. CYP71 and CYP89 genes belonged to cytochrome P450, previously reported to the highly expression by environmental stress $31,38,39$. Pathways related to leaf morphogenesis down-regulated significantly in drought stress at 9 day after start of experiment (Table. 2). In the current study TCP4 and TCP2 down-regulated, this indicated that walnut under drought conditions changes of leaf morphology. Previous research report that under drought stress leaf morphogenesis regulated by miR319 targets to the gene family of TCP transcription factors ${ }^{40}$. Overall in the present study, more drought tolerance pathways were activated at 18 days than at 9 days after the start of the experiment. The important pathways including up and down-regulated showed in Fig. 6. Biosynthesis and catabolism of $A B A$ is essential for drought tolerance in plants, including stress-responsive gene expression, metabolic changes and stomatal closure ${ }^{41}$. ABA biosynthesis pathway enriched significantly in S18 than C18, list of gene expression in this pathway given in Table. 3. NCED6 encodes 9-cis-epoxycarotenoid dioxygenase, a key enzyme in the biosynthesis of abscisic acid ${ }^{42}$. ABA4 is essential for neoxanthin biosynthesis that intermediary step in abscisic acid biosynthesis ${ }^{43}$. Other genes play a key role in the synthesis and transport of ABA including ARIA ${ }^{44}, A H G 1{ }^{45}, A F P 2{ }^{46}$ and XERICO ${ }^{47}$. Other pathway that enriched significant in $\mathrm{S} 18$ is regulation of stomatal opening, actually, the role of this pathway is to close the stomata opening in collaboration with the ABA biosynthesis pathway in order to prevent massive water loss from the plants ${ }^{8}$. One of the genes that encodes a calmodulin-binding protein involved plays key roles in stomatal movement is IQM1 ${ }^{48}$. Response to oxidative stress, carbohydrate metabolism and secondary metabolite biosynthesis pathways activated in S9 and S18, and gene name with full change given in Table 2 and 3. These findings suggest that walnut activate certain pathways in early drought, but at long droughts, different gene expressed (compare Table 2 and 3). For example, compared to its transcript levels in C18, HSPRO2 gene up-regulated in S18 group, but not expression in S9. In Arabidopsis transgenic overexpressing of HSPRO2 gene showed that high tolerance to oxidative stress than the wild type ${ }^{49}$. In the other study researcher reported that Arabidopsis transgenic with overexpression of GATL10 gene have increased galactionol and raffinose levels than the wild type plants ${ }^{50}$. Increasing the concentration of these two substances protects salicylic acid against the free oxygen radicals ${ }^{51}$. Interestingly, 'response to salt stress' pathway enriched significantly in the S18 than C18 (Fig. $5 a)$. Several paper reported that there are similarities responses between salinity and drought in plants ${ }^{51,52}$. As shown in Fig. 5b ATP biosynthesis pathway down-regulated under drought stress in S18 group. This finding is in agreement with ${ }^{53}$ report that the tricarboxylic acid (TCA) cycle and ATP biosynthesis are negatively affected under drought stress. Transcription factors such as WRKY, NAC, BZIP, MYB, ERF and ERF family members played vital roles in drought stress tolerance by regulation of 
hormone metabolism and hormone signalling transduction pathways ${ }^{32,54}$. Several groups of transcription factors have been identified under drought stress in S18 than C18 (Table 6). MYB transcription factors has different roles in plants. For instance, some of them are expressed in the stomatal and affect the movements of the stomatal, some also affect flowering, cell wall, cuticle, suberines, flavonoids and abiotic stress ${ }^{55}$. MYB 78 and MYB 2 key role in drought tolerance in plants ${ }^{55}$, MYB 15 closure the stomatal in plants ${ }^{56}$ and another study reported the role of MYB3R1 in stomatal movements under drought stress in Arabidopsis ${ }^{57}$. NAC transcription factors is the second group that identified in the present study. This transcription factor usually acts specifically on plants and even tissues. In terms of specificity, some NAC are expressed in specific tissues, some in specific developmental stages, and some in response to specific stresses ${ }^{58}$. For example 40 types of this transcription factor in rice ${ }^{59}$ and 38 types in soybeans ${ }^{60}$ have increased expression under drought stress conditions. Also, in other study reported that, Arabidopsis transgenic with NAC 072 high tolerance to drought stress that wild type ${ }^{61}$. WRKY are third group of transcription factors that have increased expression in the present study (Table 6). There have been several reports of WRKY transcription factor roles in drought stress. These roles include the transfer of ABA hormones, increased plant susceptibility to oxidative stress, the transfer of sugars and the transfer of jasmonic acid ${ }^{62}$. For example, reported WRKY 6 play a role in transfer of ABA in plants ${ }^{63}$. ERF types were the fourth group of transcription factors that increased expression in S18 groups (Table. 6). Ethylene Response Transcription Factors (ERFs) are a large group of plant transcription factors that are involved regulation of gene expression in responses to plant hormones and responses to biotic and abiotic stresses ${ }^{64,65}$. ERF4 transcription factor play a positive role in the growth and tolerance to drought stress in rice ${ }^{66}$. Also, reported that ERF1 transcription factor was directly involved in tolerance to drought stress in arabidopsis. This transcription factor is involved in the transfer of the ethylene, abscisic acid, and jasmonic acid ${ }^{67}$.

\section{Conclusion}

The present study was designed to determine the transcriptomic response of leaf against drought stress in walnut. We identified 921 DEGs between C9-S9, 1035 DEGs between C18-S18 and 841 DEGs between S9-S18 groups. Our results for GO enrichment showed that in day 9th of drought stress exposure, Oxidative stress, Carbohydrate metabolism, Cell wall macromolecule catabolism and Secondary metabolite biosynthesis pathways were up-regulated and Leaf morphogenesis, Polysaccharide biosynthesis and Acetyl-coA biosynthesis pathways were down-regulated in drought stress conditions. Also, day 18th of drought stress exposure Response to drought stress, Abscisic acid, Secondary metabolite biosynthesis, Response to oxidative stress and Regulation of stomatal opening pathways were up-regulated and ATP biosynthesis, vacuole organization and starch catabolism pathways were down-regulated in drought stress groups. We also identified several groups of transcription factors associated with drought tolerance in walnut. A schematic model is represented for the transcriptional responses associated with the drought tolerance in walnut is shown in Fig. 9. This research extends our 
knowledge regarding the mechanisms that are involved in drought stress responses of walnut and provide useful genomic information for future studies.

\section{Methods}

\section{Plant material, growth conditions and drought stress}

Plant material and growth conditions in the current study described in the recent publication ${ }^{67}$. Briefly. Two-year old clonally propagated walnut cv. 'Chandler' were used in present study. These plants were grown in two-liter pots containing peat and perlite $(1 / 1, v / v)$ and kept in the greenhouse under control conditions (with $16 \mathrm{~h}$ light/8 $\mathrm{h}$ dark photoperiod). To apply drought stress to the chandler cultivar, eighteen pots were selected and divided in two groups including control and drought-exposed plants (i.e. nine pots per each group) for a total of three replicates, with three pots each. Control plants were watered once every two days with distilled water and drought stress was applied by water withholding. Finally, leaves samples were harvested from control and stress treatment at 9 and 18 days after starting treatments. The leaf samples were frozen in liquid $\mathrm{N}_{2}$ and stored at $-80^{\circ} \mathrm{C}$ for subsequent uses.

\section{Physiological Measurements}

\section{Relative water content (RWC)}

Fully developed young leaves were taken at $9^{\text {th }}$ and $18^{\text {th }} \mathrm{d}$ after water holding from both control and water deficit-exposed plants between 9-10 am and RWC was calculated according the formula; (FW DW) $/(T W-D W) \times 100$ where FW is fresh weight, DW is dry weight (after drying disks in $70{ }^{\circ} \mathrm{C}$ for $48 \mathrm{~h}$ ) and TW is turgid weight (adding approximately $3 \mathrm{ml}$ of distilled water to disks for $4 \mathrm{~h}$ in room temperature).

\section{Leaf chlorophyll content}

Each treatment had five replicates, and five disks $\left(10 \mathrm{~mm}^{2}\right)$ were used for each replicate. SPAD index (leaf chlorophyll content) was estimated nondestructively for five leaves per treatment, using a portable SPAD502 chlorophyll meter (Minolta Corp., Ramsey, NJ, USA).

\section{Membrane stability index}

Membrane stability index percentage was calculated in 20 leaf discs from young leaves using equation $\mathrm{MSI}=\left(1-\mathrm{C}_{1} / \mathrm{C}_{2}\right) \times 100$ where $\mathrm{C}_{1}$ and $\mathrm{C}_{2}$ are electrical conductivity were measured before and after leaf discs incubated at $40^{\circ} \mathrm{C}$ according to the method described by 52 .

\section{Chlorophyll Fluorescence Measurements}


Young, fully developed leaves of both plants (stress and control) were used to assess maximum quantum efficiency of PSII (Fv/Fm) using a fluorometer (Fluorcam FC 1000-H, Photon Systems Instruments, PSI, Czech Republic). For this purpose, the plants with intact leaves were kept in dark for 20 min before measurements. After dark adaptation, plants were immediately used to measure Fv/Fm according to the methods described by ${ }^{68}$. The polyphasic Chlorophyll a fluorescence (OJIP) transients were measured in the young expanded leaves using Fluorpen FP 100-MAX (Photon Systems Instruments, Drasov, Czech Republic). OJIP was employed to study biophysical and phenomenological status of electron transport chain of photosynthetic apparatus ${ }^{69}$. To do this, after 20 min dark adaptation, protocol for OJIP transient were run. This protocol was used to track fluorescence emission at $50 \mu \mathrm{s}(\mathrm{F} 50 \mu \mathrm{s}$, considered as the minimum fluorescence F0), 2 ms (J-step, FJ), 60 ms (I-step, FI) and $300 \mathrm{~ms} \mathrm{(Fm).} \mathrm{FGluorpen} \mathrm{software} \mathrm{was}$ used for data extraction from the original measurements.

\section{Molecular Study}

\section{RNA extraction and sequencing}

Young leaves samples were taken from the control and drought stress plants and immediately frozen in liquid nitrogen and stored at $-80^{\circ} \mathrm{C}$ till use. Total RNA was isolated from 11 samples using Qiagen RNeasy mini kit (Qiagen) according to the manufacturer's instructions. NanoDrop (Thermo Scientific ${ }^{\text {TM }}$ NanoDrop 2000) was applied to measure the concentration of RNA. Ratio of $28 \mathrm{~S}$ to $18 \mathrm{~S}$ ribosomal RNA bands (28S/18S ratio) was checked on $1 \%$ agarose gels. The samples with $28 \mathrm{~s} / 18 \mathrm{~s}$ ratio $>1.8$ and an OD 260/280 ratio greater than 1.9 were sent to BGI Co. in China for sequencing. The integrity and quality of total RNA were examined by the RIN algorithm of the Agilent Bio analyzer 2100 system (Agilent RNA 6000 Nano Kit, Agilent, Cat No.5067-1511) in BGI Co and the samples with RIN > 7 were selected for CDNA library construction. All the libraries were sequenced on an Illumina Hiseq 2000 platform, and $150 \mathrm{bp}$ paired-end reads were generated. All the reads were checked for data quality using FASTQC (v0.11.5) (http://www.bioinformatics.bbsrc.ac.uk/projects/fastqc/). Clean reads were obtained by removing reads containing adapters and low-quality basses/reads $(Q<20)$ using Trimmomatic software (Version 0.36). Then, all clean reads were error corrected with Rcorrector software (version 1.0.1), which is a Kmer-based error correction of RNA-Seq reads ${ }^{70}$.

\section{De Novo Assembly And Functional Annotation}

For de novo assembly, we used from five different assemblers, namely, Bridger (version r2014-12-01) ${ }^{35}$, BinPacker (version 1.0.0) ${ }^{71}$, SOAPdenovo-Trans (version 1.03) ${ }^{72}$, Trinity (version 2.0.6) ${ }^{73}$ and rnaSPAdes (version 3.11.1) ${ }^{74}$. Finally, all transcriptome assemblies that had been assembled by two merged assemblers namely, EvidentialGene version 2013.07.27; (http://arthropods.eugenes.org/EvidentialGene/) and the Transfuse v0.5.0 (https://github.com/cboursnell/transfuse) into a single assembly. After assembly quality assessment the 
best assembler was chosen ${ }^{67}$. The clean reads were assembled with EvidentialGene version 2013.07.27; (http://arthropods.eugenes.org/EvidentialGene/) software using the default setting. EvidentialGene pipeline enables us to reduce the complexity of the de novo transcriptome assemblies using remove the highly similar transcripts, sequence fragments and transcripts with low coding potential. In the following, quality of assembly was evaluated by N50 length, reads mapping back to transcriptome (RMBT) and BUSCO (Benchmarking Universal Single-Copy Orthologs, version 2.0.1). TransDecoder (version v2.0.1, available at http://transdecoder.github.io) was applied to translate the assembled transcripts to putative coding sequences. Assembled transcripts were aligned against the following databases, National Centre for Biotechnology Information (NCBI) Non-Redundant database and Nucleotide Collection (Nr/Nt), Pfam, Swiss-Prot, Gene Ontology (GO), Kyoto Encyclopedia of Genes and Genomes (KEGG) and the Karyotic Orthologous Groups (KOG) with an Evalue cutoff $1 \mathrm{E} \otimes 5$.

\section{Differential Gene Expression Analysis}

First, reads were mapped to the final assembled transcriptome using Bowtie (version 1.1.2) with default parameters. RSEM software (version 1.3.2) was used to quantify transcript expression in all samples ${ }^{75}$. Finally, edgeR package (version 3.26.4) of R software was used to perform count-based differential expression analysis. An false discovery rate (FDR) $<0.001$ and a normalized fold change $\geq 2$ were set as the thresholds to identify DEGs. Overall, three pair-wise comparisons were considered including control (C9) and drought stress (S9) in 9-day, control (C18) and drought stress (S18) in 18-day and drought stress in 9 (S9) and 18 (S18) day after beginning the experiment.

The GOseq package of R software was used to identify significant GO terms. Also, Web Gene Ontology Annotation Plot (WEGO) was applied to functional categorization of all the annotated transcripts ${ }^{76}$. The GO category enrichment analysis for DEGs was achieved via REVIGO (http://revigo.irb.hr/). Calculation and visualization of Venn diagrams were conducted through online software, http://bioinformatics.psb.ugent.be/webtools/Venn/. Drought tolerance pathway construction and visualization was achieved through Pathvisio3 software ${ }^{77}$.

\section{Validation Of Rna-seq Results By Rt-pcr Analysis}

A total of six DEGs (four up-regulated and two down-regulated) were randomly selected to validate the RNA-Seq results using RT-PCR. In brief, total RNA was extracted using TRIzol reagent (Invitrogen, USA). Then, $2 \mu \mathrm{g}$ of total RNA was used for first-strand cDNA synthesis using the Thermo Scientific kit (K1621, USA) according to the manufacturer's protocol. Primer sequences were designed using the Oligocalculator and primer3 programs (Table S3). The walnut Actin2 was used as a housekeeping gene to verify the expression results of sequencing. RT-PCR was conducted using a HIFI SYBR Green Master Mix (Mabnateb, Iran). The cycling instructions was $15 \mathrm{~min}$ at $95^{\circ} \mathrm{C}$, followed by 40 cycle of $15 \mathrm{~s}$ at $95^{\circ} \mathrm{C}$ for denaturation. $60 \mathrm{~s}$, at $60^{\circ} \mathrm{C}$ for annealing and $120 \mathrm{~s}$ at $60^{\circ} \mathrm{C}$ for extension. All reactions were done 
with triple biological replicates and two technical replicates. Relative expression of each gene was calculated using $2^{\Delta \Delta \mathrm{CT}}$ method.

\section{Declarations}

\section{Acknowledgments}

The authors appreciate University of Jiroft, University of Tehran, Iran National Science Foundation (INSF) grant number; 960805 and Center of Excellence of Walnut Improvement and Technology of Iran for their supports.

\section{Author contributions}

M.S-H: Investigation and manuscript writing, MR.B.: Data curation, Project administration and software, N.B.: Visualization, Funding acquisition and review \& editing manuscript, M.T.: real-time analysis and review \& editing manuscript, S.A.: physiological analysis and review \& editing manuscript, and K.V.: Formal analysis, review \& editing manuscript. All authors have read and agreed to the published version of the manuscript.

\section{Competing interests}

The authors declare no competing interests.

\section{References}

1. Vahdati, K. et al. Advances in Persian Walnut (Juglans regia L.) Breeding Strategies. in Advances in Plant Breeding Strategies: Nut and Beverage Crops: Volume 4 (eds. Al-Khayri, J. M., Jain, S. M. \& Johnson, D. V) 401-472 (Springer International Publishing, 2019). doi:10.1007/978-3-030-231125_11.

2. Vahdati, K. et al. Screening for Drought-tolerant Genotypes of Persian Walnuts (Juglans regia L.) During Seed Germination. HortScience horts44, 1815-1819.

3. Famula, R. A., Richards, J. H., Famula, T. R. \& Neale, D. B. Association genetics of carbon isotope discrimination and leaf morphology in a breeding population of Juglans regia L. Tree Genet. Genomes15, 6 (2018).

4. Arab, M. M. et al. Combining phenotype, genotype, and environment to uncover genetic components underlying water use efficiency in Persian walnut. J. Exp. Bot.71, 1107-1127 (2020).

5. Nakashima, K., Ito, Y. \& Yamaguchi-Shinozaki, K. Transcriptional Regulatory Networks in Response to Abiotic Stresses in Arabidopsis and Grasses. Plant Physiol.149, 88 LP - 95 (2009).

6. Gugger, P. F., Peñaloza-Ramírez, J., Wright, J. \& Sork, V. Whole-transcriptome response to water stress in a California endemic oak, Quercus lobata. Tree Physiol.37, 63R_644 (2017). 
7. Basu, S., Ramegowda, V., Kumar, A. \& Pereira, A. Plant adaptation to drought stress. F1000Research5, (2016).

8. Daszkowska-Golec, A. \& Szarejko, I. Open or close the gate-stomata action under the control of phytohormones in drought stress conditions. Front. Plant Sci.4, 138 (2013).

9. Fracasso, A., Trindade, L. M. \& Amaducci, S. Drought stress tolerance strategies revealed by RNA-Seq in two sorghum genotypes with contrasting WUE. BMC Plant Biol.16, 115 (2016).

10. Arms, E. M., Yan, Z. \& St Clair, D. A. Differential transcriptional regulation in roots of tomato nearisogenic lines in response to rapid-onset water stress. Front. Plant Sci.8, 166 (2017).

11. Zhang, F. et al. Genetic regulation of salt stress tolerance revealed by RNA-Seq in cotton diploid wild species, Gossypium davidsonii. Sci. Rep.6, 20582 (2016).

12. Dong, B. et al. Transcriptome analysis of the tea oil camellia (Camellia oleifera) reveals candidate drought stress genes. PLoS One12, e0181835-e0181835 (2017).

13. Li, H., Lin, J., Yang, Q.-S., Li, X.-G. \& Chang, Y.-H. Comprehensive analysis of differentially expressed genes under salt stress in pear (Pyrus betulaefolia) using RNA-Seq. Plant Growth Regul.82, 409-420 (2017).

14. Ksouri, N., Jiménez, S., Wells, C. E., Contreras-Moreira, B. \& Gogorcena, Y. Transcriptional responses in root and leaf of Prunus persica under drought stress using RNA sequencing. Front. Plant Sci.7, 1715 (2016).

15. Villar, E. et al. RNA-Seq reveals genotype-specific molecular responses to water deficit in eucalyptus. BMC Genomics12, 538 (2011).

16. Yadav, R., Lone, S. A., Gaikwad, K., Singh, N. K. \& Padaria, J. C. Transcriptome sequence analysis and mining of SSRs in Jhar Ber (Ziziphus nummularia (Burm. f.) Wight \& Arn) under drought stress. Sci. Rep.8, 1-10 (2018).

17. Naser, L., Kourosh, V., Bahman, K. \& Reza, A. Soluble sugars and proline accumulation play a role as effective indices for drought tolerance screening in Persian walnut (Juglans regia L.) during germination. Fruits65, 97-112 (2010).

18. Tyree, M. T., Cochard, H., Cruiziat, P., Sinclair, B. \& Ameglio, T. Drought-induced leaf shedding in walnut: evidence for vulnerability segmentation. Plant. Cell Environ.16, 879-882 (1993).

19. Tardieu, F., Simonneau, T. \& Muller, B. The physiological basis of drought tolerance in crop plants: a scenario-dependent probabilistic approach. Annu. Rev. Plant Biol.69, 733-759 (2018).

20. Tardieu, F., Parent, B., Caldeira, C. F. \& Welcker, C. Genetic and physiological controls of growth under water deficit. Plant Physiol.164, 1628-1635 (2014).

21. Bashir, K., Matsui, A., Rasheed, S. \& Seki, M. Recent advances in the characterization of plant transcriptomes in response to drought, salinity, heat, and cold stress. F1000Research8, (2019).

22. Lotfi, N., Soleimani, A., Vahdati, K. \& Çakmakçı, R. Comprehensive biochemical insights into the seed germination of walnut under drought stress. Sci. Hortic. (Amsterdam).250, 329-343 (2019). 
23. Heidari, L., Boroomand, N. \& Sadat-Hosseini, M. The Effect of Different Water Potentials on Seed Germination and Growth of some Persian Walnut Populations. J. Nuts10, 186-201 (2019).

24. Lawlor, D. W. \& Tezara, W. Causes of decreased photosynthetic rate and metabolic capacity in waterdeficient leaf cells: a critical evaluation of mechanisms and integration of processes. Ann. Bot.103, 561-579 (2009).

25. Wada, S., Suzuki, Y. \& Miyake, C. Photorespiration Enhances Acidification of the Thylakoid Lumen, Reduces the Plastoquinone Pool, and Contributes to the Oxidation of P700 at a Lower Partial Pressure of CO2 in Wheat Leaves. Plants9, (2020).

26. Zhou, Y., Lam, H. M. \& Zhang, J. Inhibition of photosynthesis and energy dissipation induced by water and high light stresses in rice. J. Exp. Bot.58, 1207-1217 (2007).

27. Cruz de Carvalho, M. H. Drought stress and reactive oxygen species: production, scavenging and signaling. Plant Signal. Behav.3, 156-165 (2008).

28. Zhu, Y. et al. Transcriptomic identification of drought-related genes and SSR markers in Sudan grass based on RNA-Seq. Front. Plant Sci.8, 687 (2017).

29. Kollist, H. et al. Rapid responses to abiotic stress: priming the landscape for the signal transduction network. Trends Plant Sci.24, 25-37 (2019).

30. Christmann, A., Grill, E. \& Huang, J. Hydraulic signals in long-distance signaling. Curr. Opin. Plant Biol.16, 293-300 (2013).

31. Wang, Y. et al. Transcriptional and physiological analyses of short-term Iron deficiency response in apple seedlings provide insight into the regulation involved in photosynthesis. BMC Genomics19, 112 (2018).

32. Gonçalves, L. P. et al. Rootstock-induced molecular responses associated with drought tolerance in sweet orange as revealed by RNA-Seq. BMC Genomics20, 110 (2019).

33. Liu, J., Deng, J. L. \& Tian, Y. Transcriptome sequencing of the apricot (Prunus armeniaca L.) and identification of differentially expressed genes involved in drought stress. Phytochemistry171, 112226 (2020).

34. Mittler, R. Oxidative stress, antioxidants and stress tolerance. Trends Plant Sci.7, 405-410 (2002).

35. Tsai, Y.-C., Hong, C.-Y., Liu, L.-F. \& Kao, C. H. Expression of ascorbate peroxidase and glutathione reductase in roots of rice seedlings in response to $\mathrm{NaCl}$ and $\mathrm{H} 2 \mathrm{O} 2$. J. Plant Physiol.162, 291-299 (2005).

36. Amist, N. \& Singh, N. B. The role of sugars in the regulation of environmental stress. in Plant Life Under Changing Environment 497-512 (Elsevier, 2020).

37. Niinemets, Ü. Uncovering the hidden facets of drought stress: secondary metabolites make the difference. Tree Physiol.36, 129-132 (2016).

38. Murgia, I., Tarantino, D., Soave, C. \& Morandini, P. Arabidopsis CYP82C4 expression is dependent on Fe availability and circadian rhythm, and correlates with genes involved in the early Fe deficiency response. J. Plant Physiol.168, 894-902 (2011). 
39. Bi, H. et al. Wheat drought-responsive WXPL transcription factors regulate cuticle biosynthesis genes. Plant Mol. Biol.94, 15-32 (2017).

40. Li, S. \& Zachgo, S. TCP 3 interacts with R2R3-MYB proteins, promotes flavonoid biosynthesis and negatively regulates the auxin response in A rabidopsis thaliana. Plant J.76, 901-913 (2013).

41. Seki, M., Umezawa, T., Urano, K. \& Shinozaki, K. Regulatory metabolic networks in drought stress responses. Curr. Opin. Plant Biol.10, 296-302 (2007).

42. Lefebvre, V. et al. Functional analysis of Arabidopsis NCED6 and NCED9 genes indicates that ABA synthesized in the endosperm is involved in the induction of seed dormancy. Plant J.45, 309-319 (2006).

43. Neuman, H., Galpaz, N., Cunningham Jr, F. X., Zamir, D. \& Hirschberg, J. The tomato mutation nxd1 reveals a gene necessary for neoxanthin biosynthesis and demonstrates that violaxanthin is a sufficient precursor for abscisic acid biosynthesis. Plant J.78, 80-93 (2014).

44. Lee, S., Kang, J. \& Kim, S. Y. An ARIA-interacting AP2 domain protein is a novel component of ABA signaling. Mol. Cells27, 409-416 (2009).

45. Nishimura, N. et al. ABA-Hypersensitive Germination1 encodes a protein phosphatase 2C, an essential component of abscisic acid signaling in Arabidopsis seed. Plant J.50, 935-949 (2007).

46. Lynch, T. J., Erickson, B. J., Miller, D. R. \& Finkelstein, R. R. ABI5-binding proteins (AFPs) alter transcription of ABA-induced genes via a variety of interactions with chromatin modifiers. Plant Mol. Biol.93, 403-418 (2017).

47. Ko, J., Yang, S. H. \& Han, K. Upregulation of an Arabidopsis RING-H2 gene, XERICO, confers drought tolerance through increased abscisic acid biosynthesis. Plant J.47, 343-355 (2006).

48. Zhou, Y.-P., Duan, J., Fujibe, T., Yamamoto, K. T. \& Tian, C.-E. AtIQM1, a novel calmodulin-binding protein, is involved in stomatal movement in Arabidopsis. Plant Mol. Biol.79, 333-346 (2012).

49. Luhua, S., Ciftci-Yilmaz, S., Harper, J., Cushman, J. \& Mittler, R. Enhanced tolerance to oxidative stress in transgenic Arabidopsis plants expressing proteins of unknown function. Plant Physiol.148, 280292 (2008).

50. Matthes, M. C. et al. The transcriptome of cis-jasmone-induced resistance in Arabidopsis thaliana and its role in indirect defence. Planta232, 1163-1180 (2010).

51. Nishizawa, A., Yabuta, Y. \& Shigeoka, S. Galactinol and raffinose constitute a novel function to protect plants from oxidative damage. Plant Physiol.147, 1251-1263 (2008).

52. Singh, V., Pallaghy, C. K. \& Singh, D. Phosphorus nutrition and tolerance of cotton to water stress: II. Water relations, free and bound water and leaf expansion rate. F. Crop. Res.96, 199-206 (2006).

53. Salehi-Lisar, S. Y. \& Bakhshayeshan-Agdam, H. Drought stress in plants: causes, consequences, and tolerance. in Drought Stress Tolerance in Plants, Vol 11-16 (Springer, 2016).

54. Huang, Q. et al. TaNAC29, a NAC transcription factor from wheat, enhances salt and drought tolerance in transgenic Arabidopsis. BMC Plant Biol.15, 268 (2015). 
55. Baldoni, E., Genga, A. \& Cominelli, E. Plant MYB transcription factors: their role in drought response mechanisms. Int. J. Mol. Sci.16, 15811-15851 (2015).

56. Ding, Z. et al. Transgenic expression of MYB15 confers enhanced sensitivity to abscisic acid and improved drought tolerance in Arabidopsis thaliana. J. Genet. Genomics36, 17-29 (2009).

57. Cai, H., Tian, S., Dong, H. \& Guo, C. Pleiotropic effects of TaMYB3R1 on plant development and response to osmotic stress in transgenic Arabidopsis. Gene558, 227-234 (2015).

58. Joshi, R. et al. Transcription factors and plants response to drought stress: current understanding and future directions. Front. Plant Sci.7, 1029 (2016).

59. Shao, H., Wang, H. \& Tang, X. NAC transcription factors in plant multiple abiotic stress responses: progress and prospects. Front. Plant Sci.6, 902 (2015).

60. Le, D. T. et al. Genome-wide survey and expression analysis of the plant-specific NAC transcription factor family in soybean during development and dehydration stress. DNA Res.18, 263-276 (2011).

61. Liu, G. et al. Overexpression of rice NAC gene SNAC1 improves drought and salt tolerance by enhancing root development and reducing transpiration rate in transgenic cotton. PLoS One9, e86895 (2014).

62. Bakshi, M. \& Oelmüller, R. WRKY transcription factors: Jack of many trades in plants. Plant Signal. Behav.9, e27700 (2014).

63. Shang, Y. et al. The Mg-chelatase $\mathrm{H}$ subunit of Arabidopsis antagonizes a group of WRKY transcription repressors to relieve ABA-responsive genes of inhibition. Plant Cel/22, 1909-1935 (2010).

64. Sharoni, A. M. et al. Gene structures, classification and expression models of the AP2/EREBP transcription factor family in rice. Plant cell Physiol.52, 344-360 (2011).

65. Xu, Z., Chen, M., Li, L. \& Ma, Y. Functions and application of the AP2/ERF transcription factor family in crop improvement F. J. Integr. Plant Biol.53, 570-585 (2011).

66. Zhang, H. et al. Functional analyses of ethylene response factor JERF3 with the aim of improving tolerance to drought and osmotic stress in transgenic rice. Transgenic Res.19, 809-818 (2010).

67. Sadat-Hosseini, M., Bakhtiarizadeh, M. R., Boroomand, N., Tohidfar, M. \& Vahdati, K. Combining independent de novo assemblies to optimize leaf transcriptome of Persian walnut. PLoS One15, e0232005 (2020).

68. Seifikalhor, M., Hassani, S. B. \& Aliniaeifard, S. Seed Priming by Cyanobacteria (Spirulina platensis) and Salep Gum Enhances Tolerance of Maize Plant Against Cadmium Toxicity. J. Plant Growth Regul. 1-13 (2019).

69. Strasser, R. J., Srivastava, A. \& Tsimilli-Michael, M. The fluorescence transient as a tool to characterize and screen photosynthetic samples. Probing Photosynth. Mech. Regul. Adapt. 445-483 (2000).

70. Mousavi, S. et al. De novo transcriptome assembly and comparative analysis of differentially expressed genes in Prunus dulcis Mill. in response to freezing stress. PLoS One9, e104541 (2014). 
71. Liu, J. et al. BinPacker: packing-based de novo transcriptome assembly from RNA-seq data. PLoS Comput. Biol.12, e1004772 (2016).

72. Xie, Y. et al. SOAPdenovo-Trans: de novo transcriptome assembly with short RNA-Seq reads. Bioinformatics30, 1660-1666 (2014).

73. Grabherr, M. G. et al. Trinity: reconstructing a full-length transcriptome without a genome from RNASeq data. Nat. Biotechnol.29, 644 (2011).

74. Bankevich, A. et al. SPAdes: a new genome assembly algorithm and its applications to single-cell sequencing. J. Comput. Biol.19, 455-477 (2012).

75. Li, B. \& Dewey, C. N. RSEM: accurate transcript quantification from RNA-Seq data with or without a reference genome. BMC Bioinformatics12, 323 (2011).

76. Ye, J. et al. WEGO: a web tool for plotting GO annotations. Nucleic Acids Res.34, W293-W297 (2006).

77. Kutmon, M. et al. PathVisio 3: an extendable pathway analysis toolbox. PLoS Comput Bio/11, e1004085 (2015).

\section{Figures}



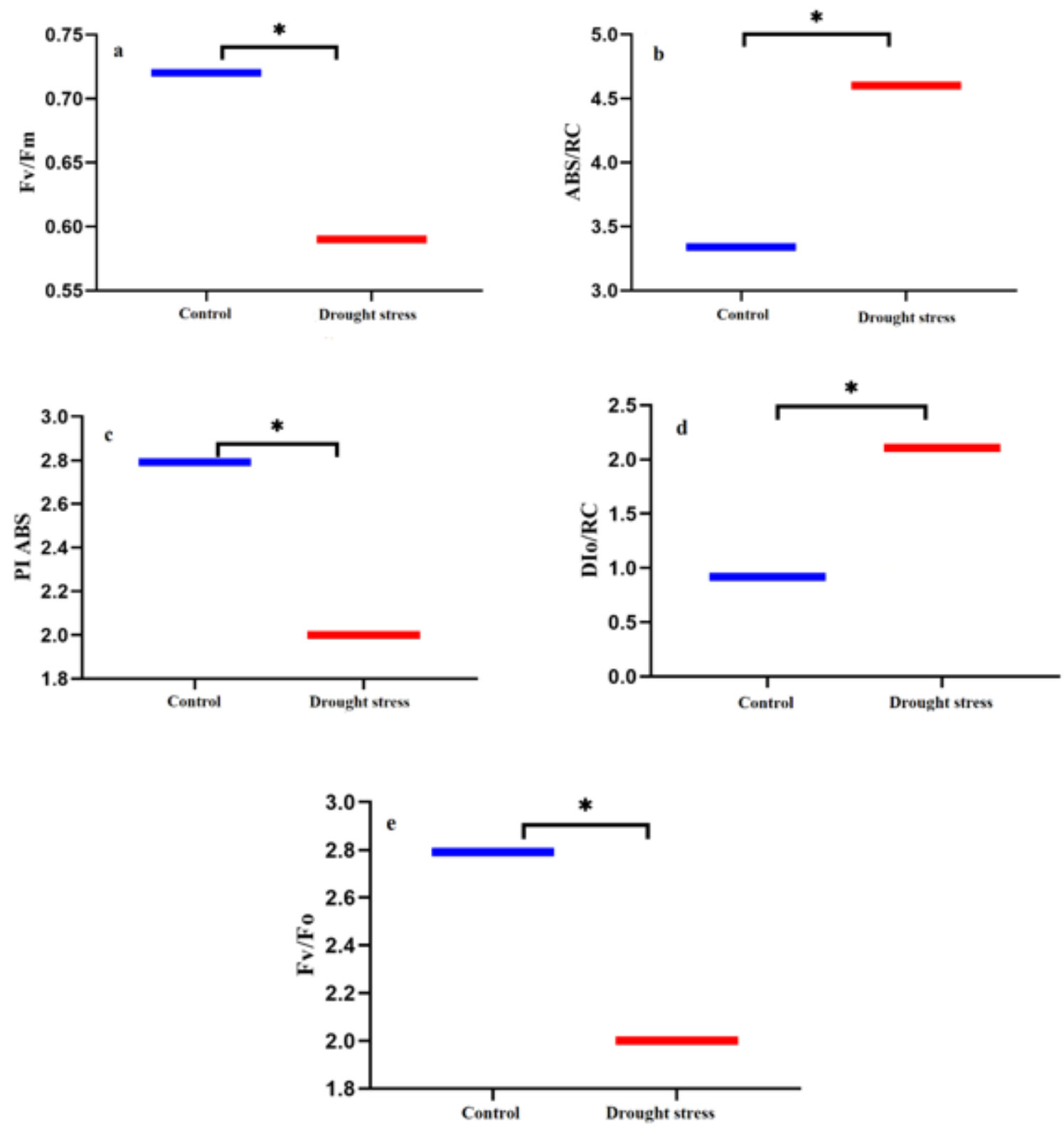

\section{Figure 1}

Effect of drought stress at 18th d on maximum quantum yield of photosystem II (Fv/Fm) (a), specific energy fluxes per reaction center (RC) for energy absorption (ABS/RC) (b), performance index on absorption basis (Pi-abs) (c), dissipated energy flux (DIO/RC) (d) and oxygen evolving complex activity $(\mathrm{fv} / \mathrm{fo})(\mathrm{e})$ in walnut. 


\section{KEGG Classification}

Environmental adaptation

Aging

817

Development

Sensory system

Nervous system

Excretory system

Digestive system

Circulatory system

Endocrine system

Immune system

Cell motility

Cellular community - prokaryotes

Cellular community - eukaryotes

Cell growth and death

Transport and catabolism

Signaling molecules and interaction

Signal transduction

Membrane transport

Replication and repair

Folding, sorting and degradation

Translation

Transcription

Xenobiotics biodegradation and metabolism

Biosyntesis of other secondary metabolites

Terpenoid backbone biosynthesis

Metabolism of cofactors and vitamins

Glican biosyntesis and metabolism

Metabolism of other amino acid

Amino acid metabolism

Nucleotid metabolism

Lipid metabolism

Energy metabolism

Carbohydrate metabolism

\begin{tabular}{l}
\hline 817 \\
\hline 347 \\
\hline 101 \\
$\square 108$ \\
\hline \\
$\square 170$ \\
$\square 256$ \\
$\square 149$ \\
\hline \\
\hline \\
\hline 119 \\
\hline
\end{tabular}

\section{7}

295

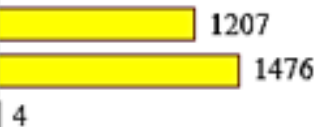

3847

\section{8}

476

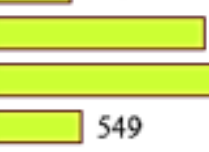

1303

1458

398

561

341

554

332

452

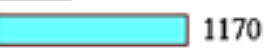

306

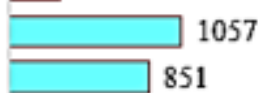

851

2028

$3000 \quad 4000$

A

B

C

D

E

$\begin{array}{llllll}0 & 1000 & 2000 & 3000 & 4000 & 5000\end{array}$

Figure 2

KEGG classification maps in walnut transcriptome: A, Organismal Systems B, Cellular Processes; C, Environmental Information Processing; D, Genetic Information Processing; and E, Metabolism. 


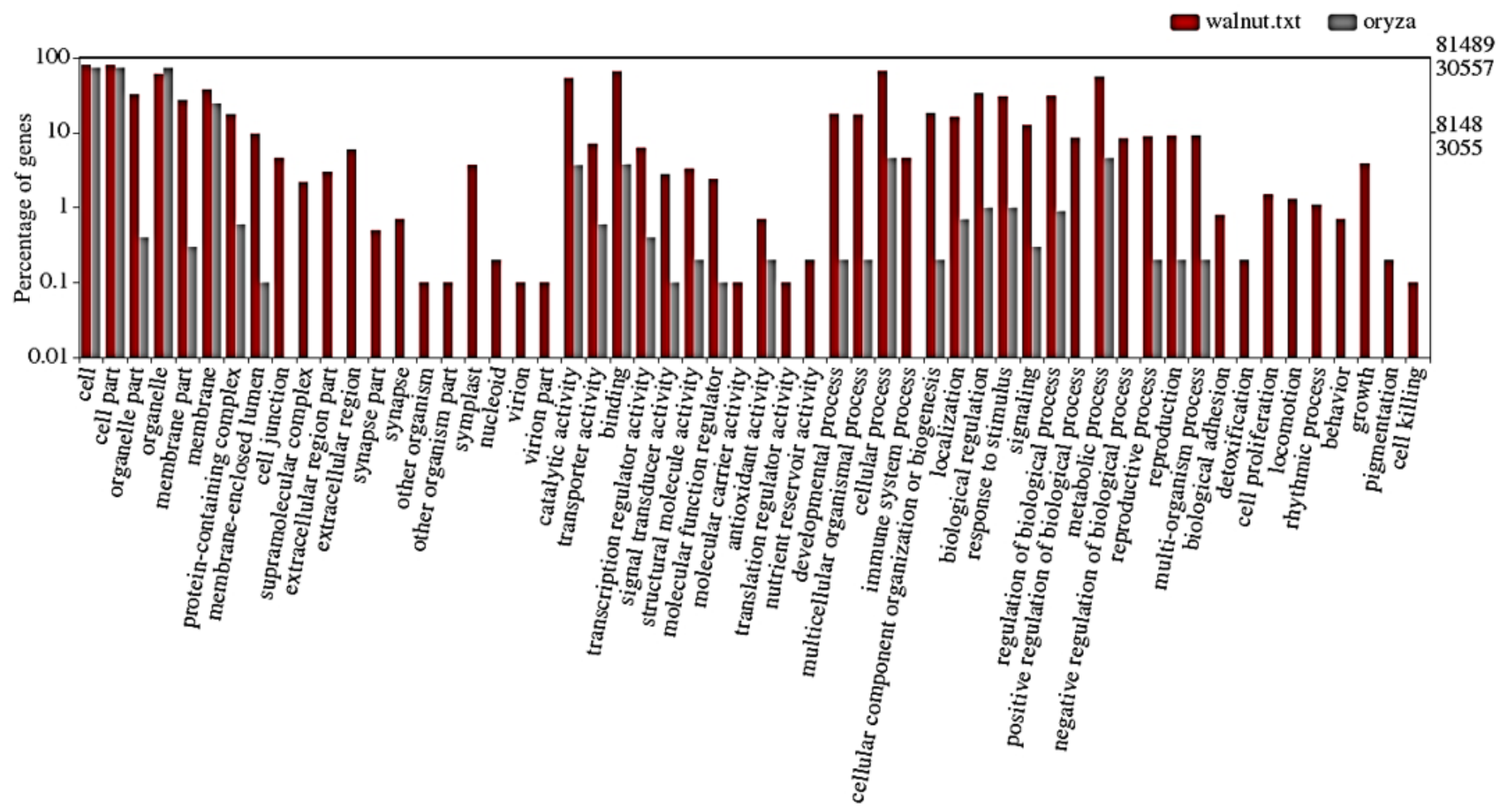

Figure 3

GO classification of the assembled transcripts in walnut. The Y-axis shows the percentage of genes in each sub-category 

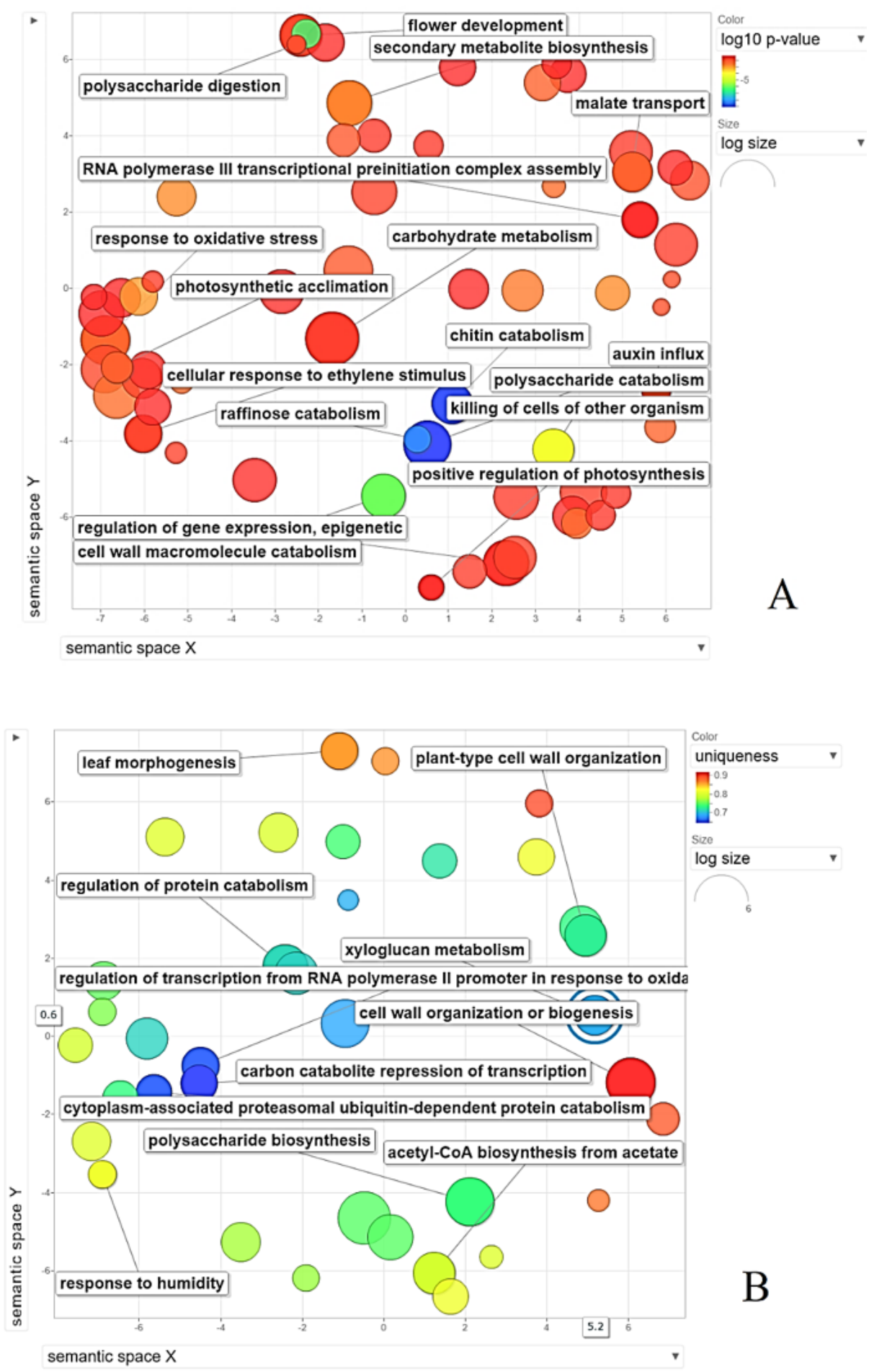

\section{Figure 4}

Results of functional enrichment analysis A) Results of the enrichment analysis for up-regulated genes under drought stress than control in 9th $d$ and $B$ ) Results of the enrichment analysis for down-regulated genes under drought stress than control in 9th $\mathrm{d}$. Circles depicted by filled color show significantly enriched GO terms with log10 p-value $<0.05$. The color and the size of bubbles show the $p$-value (legend 
in upper right-hand corner) and the frequency of the GO term in the underlying GOA database in REVIGO analysis, respectively (bubbles of more general terms are larger).

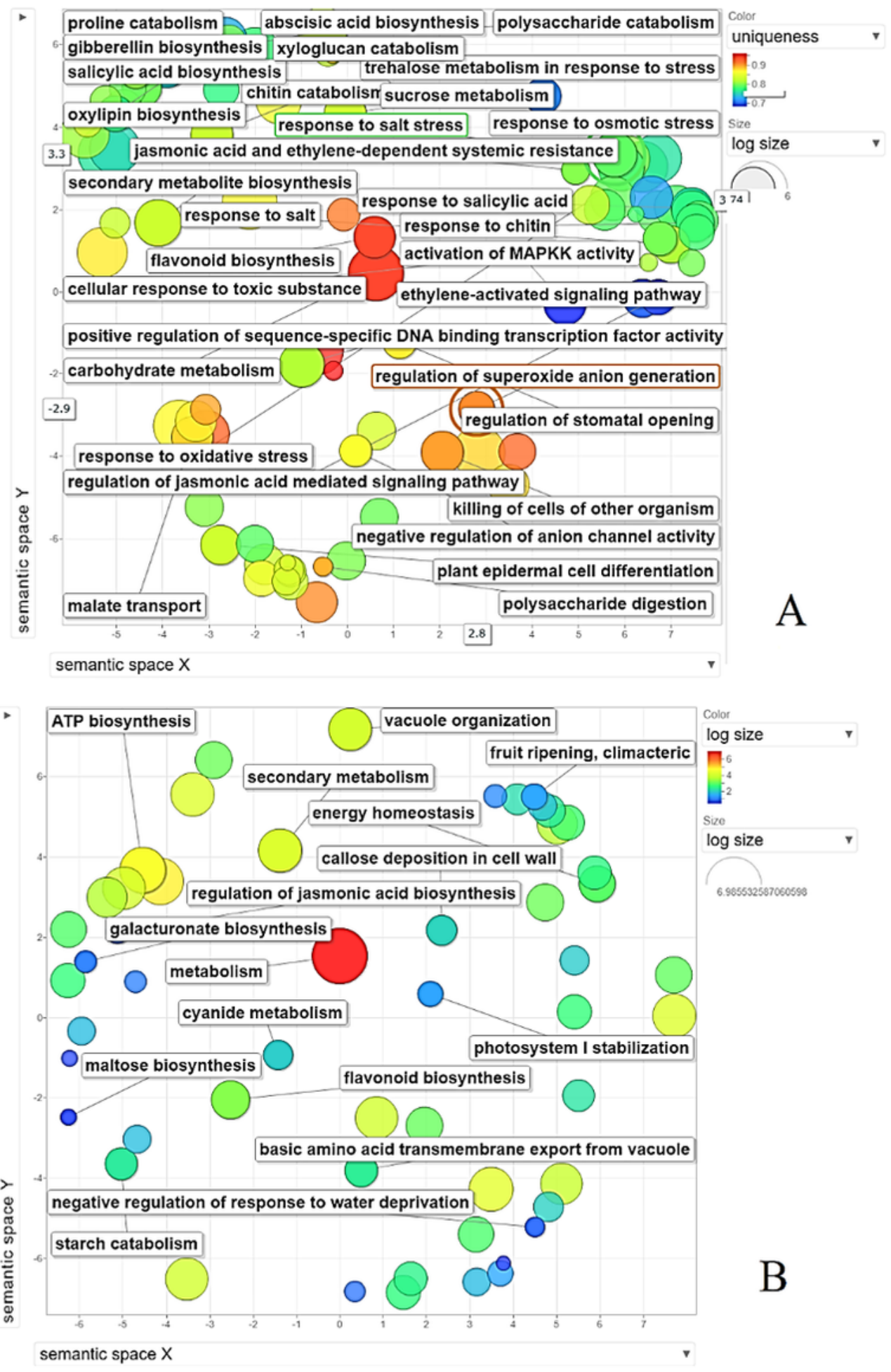

\section{Figure 5}

Results of functional enrichment analysis A) Results of the enrichment analysis for up-regulated genes under drought stress than control in 18th $d$ and B) Results of the enrichment analysis for down-regulated genes under drought stress than control in 18th $\mathrm{d}$. Circles depicted by filled color show significantly 
enriched GO terms with log10 p-value $<0.05$. The color and the size of bubbles show the $p$-value (legend in upper right-hand corner) and the frequency of the GO term in the underlying GOA database in REVIGO analysis, respectively (bubbles of more general terms are larger).
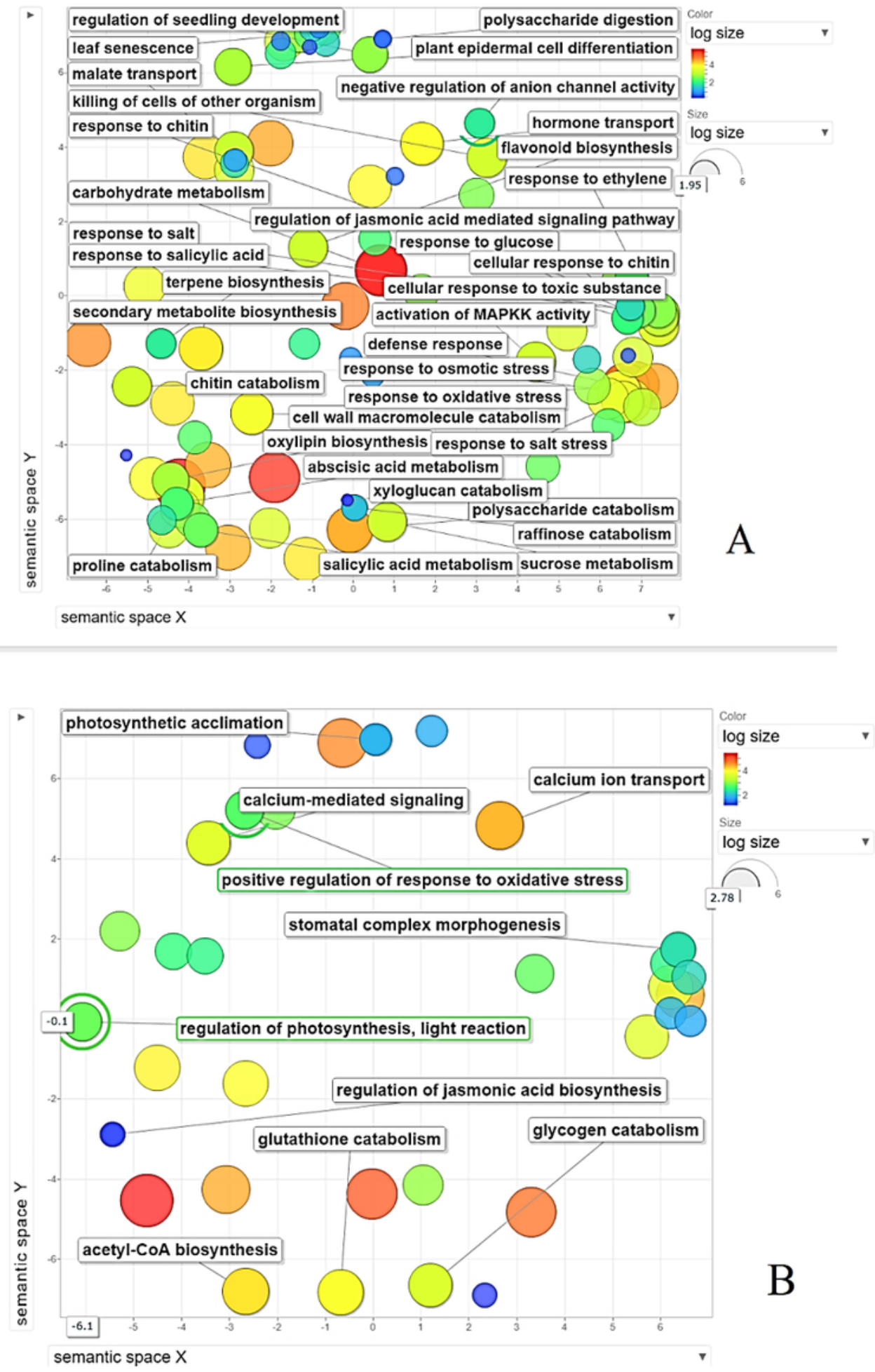

Figure 6

Results of functional enrichment analysis. A) Results of the enrichment analysis for up-regulated genes under drought stress 18th than 9th $d$ and B) Results of the enrichment analysis for down-regulated genes 
under drought stress 18th than 9th $\mathrm{d}$. Circles depicted by filled color show significantly enriched $\mathrm{GO}$ terms with $\log 10 p$-value $<0.05$. The color and the size of bubbles show the p-value (legend in upper right-hand corner) and the frequency of the GO term in the underlying GOA database in REVIGO analysis, respectively (bubbles of more general terms are larger).
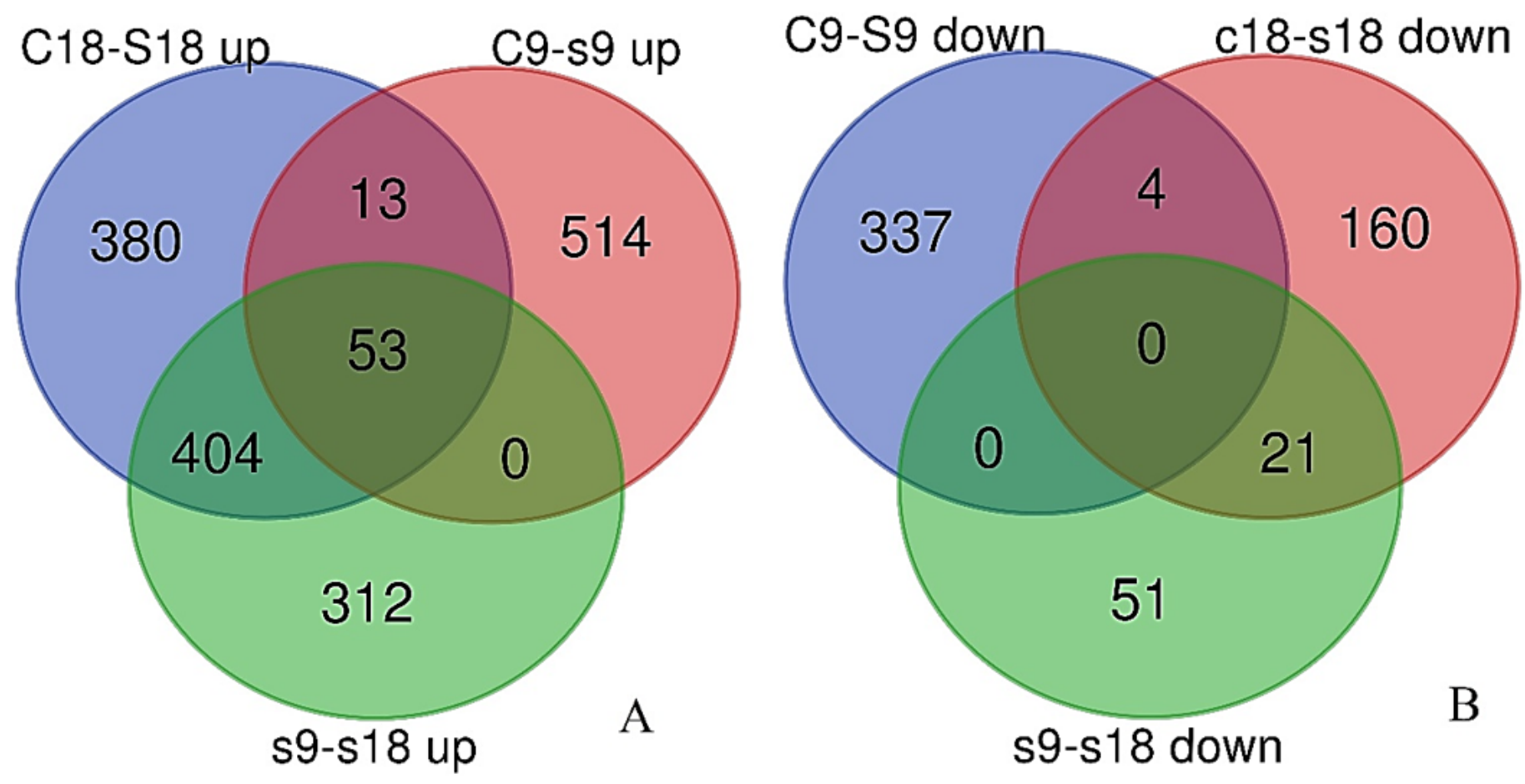

\section{Figure 7}

Venn diagrams representing common and unique up-regulated (a) and down-regulated DEGs in the three comparisons (b). 


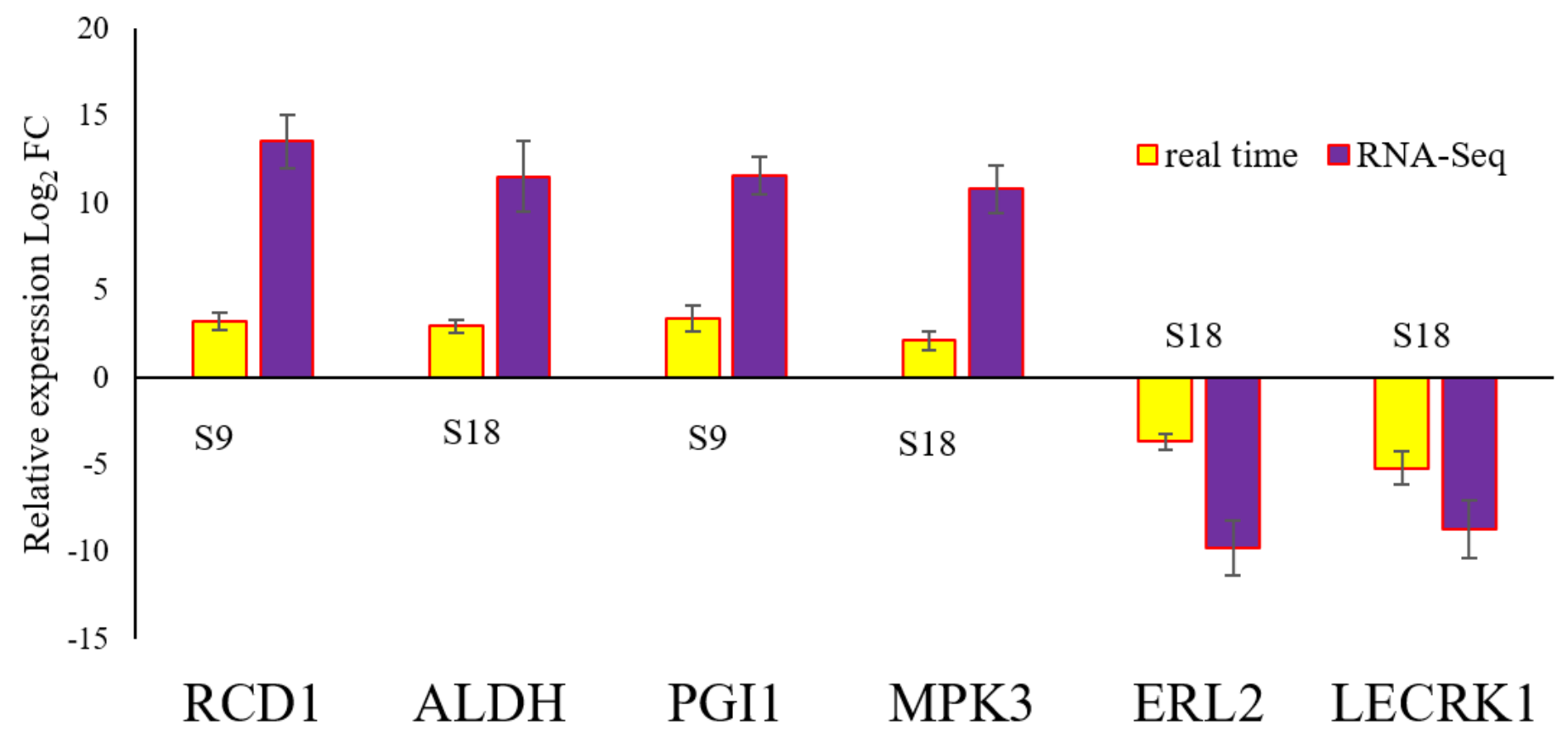

Figure 8

Comparison of the expression pattern achieved by RT-PCR and RNA-Seq. 


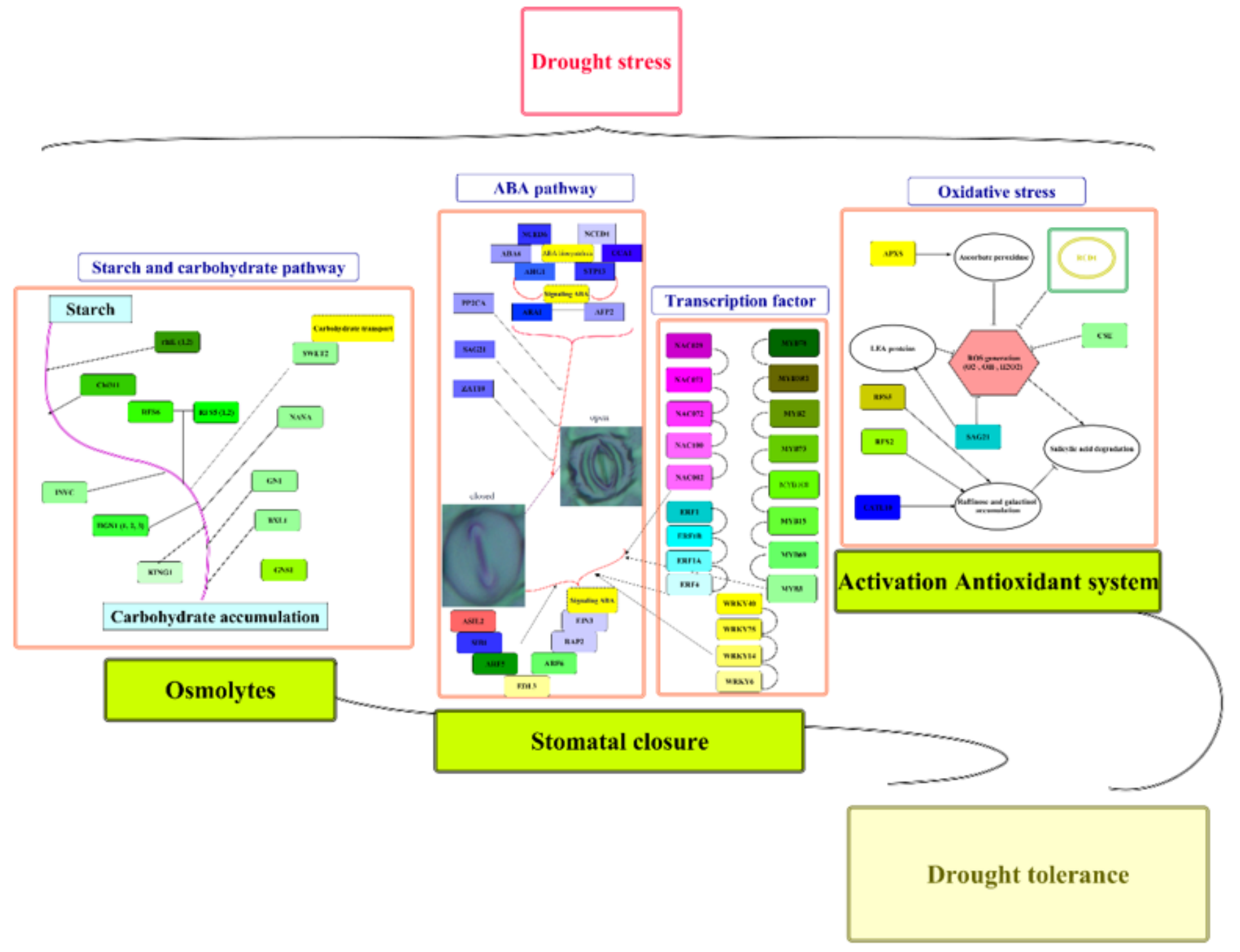

\section{Figure 9}

A Schematic overview of the activation of the pathways and genes in response to drought stress in walnut.

\section{Supplementary Files}

This is a list of supplementary files associated with this preprint. Click to download.

- TableS1new.docx 